\title{
ANNALES \\ POLONICI MATHEMATICI
}

$90.1(2007)$

\section{Monotone iteration for infinite systems of parabolic equations with functional dependence}

\author{
by Anna PudeŁko (Kraków)
}

\begin{abstract}
We consider the initial value problem for an infinite system of differentialfunctional equations of parabolic type. General operators of parabolic type of second order with variable coefficients are considered and the system is weakly coupled. The solutions are obtained by the monotone iterative method. We prove theorems on weak partial differential-functional inequalities as well the existence and uniqueness theorems in the class of continuous bounded functions and in the class of functions satisfying a certain growth condition.
\end{abstract}

1. Introduction. Let $T>0$ and $\Omega=\left\{(t, x): t \in(0, T], x \in \mathbb{R}^{m}\right\}$. Let $S$ be an infinite set of indices. Let $B(S)$ be the space of bounded mappings $v: S \ni i \mapsto v^{i} \in \mathbb{R}$ endowed with the supremum norm

$$
\|v\|_{B(S)}:=\sup \left\{\left|v^{i}\right|: i \in S\right\} .
$$

For every nonempty set $X \subset \mathbb{R}^{m}$ we denote by $C_{S}(X)$ the space of mappings

$$
w: X \ni x \mapsto w(x) \in B(S), \quad \text { where } \quad w(x): S \ni i \mapsto w^{i}(x) \in \mathbb{R},
$$

and the functions $w^{i}$ are continuous in $X$. For $w$ we use the notation $w=$ $\left\{w^{i}\right\}_{i \in S}$ as well.

Let $f=\left\{f^{i}\right\}_{i \in S}$ and $\varphi=\left\{\varphi^{i}\right\}_{i \in S}$ be given,

$$
\begin{gathered}
f^{i}: \bar{\Omega} \times B(S) \times C_{S}(\bar{\Omega}) \rightarrow \mathbb{R}, \\
\varphi^{i}: \mathbb{R}^{m} \rightarrow \mathbb{R}, \quad i \in S .
\end{gathered}
$$

Let $u=\left\{u^{i}\right\}_{i \in S}$ where each $u^{i}$ is an unknown function of the variables

2000 Mathematics Subject Classification: Primary 35K15; Secondary 35K55, 35R10, $35 \mathrm{R} 45$.

Key words and phrases: infinite systems, parabolic differential-functional equations, Cauchy problem, monotone iterative method, differential inequality.

This work is partly supported by local Grant No. 11.420.04. 
$(t, x)=\left(t, x_{1}, \ldots, x_{m}\right)$, and set

$$
\mathcal{F}^{i}:=\frac{\partial}{\partial t}-\mathcal{A}^{i}, \quad \mathcal{A}^{i}:=\sum_{j, k=1}^{m} a_{j k}^{i}(t, x) \frac{\partial^{2}}{\partial x_{j} \partial x_{k}}+\sum_{j=1}^{m} b_{j}^{i}(t, x) \frac{\partial}{\partial x_{j}}+c^{i}(t, x) .
$$

We consider an infinite system of weakly coupled $\left(^{1}\right)$ semilinear parabolic equations of reaction-diffusion-convection type of the form

$$
\mathcal{F}^{i}\left[u^{i}\right](t, x)=f^{i}(t, x, u(t, x), u), \quad i \in S,
$$

together with the initial condition

$$
u(0, x)=\varphi(x) \text { for } \quad x \in \mathbb{R}^{m} .
$$

This paper is a continuation of the author's study of certain systems of parabolic equations (cf. [9], [10]). The paper extends classical results on this subject in two directions: differential-functional equations are considered and the systems consist of infinitely many weakly coupled equations. Our aim is to prove the existence and uniqueness of a solution for system (1) with the initial condition (2). To obtain the solution we apply the so-called monotone iterative method (cf. [8]).

We construct two sequences which approximate the desired solution uniformly and monotonically.

In this approach we have to impose certain conditions, namely the monotonicity of the reaction functions in the last two variables. We also assume the existence of a pair of sub- and supersolutions (so-called lower and upper functions, respectively) for the problem in question.

These assumptions are not typical of existence theorems but the method of sub- and supersolutions, coupled with the monotone iterative technique, provides an effective and flexible mechanism that ensures theoretical as well as constructive existence results for nonlinear problems (cf. [7]). The lower and upper functions serve as bounds for solutions which are improved by a monotone iterative process.

We use some results on differential inequalities to show that sequences obtained by monotone iteration consist of sub- and supersolutions, as well as to get their uniform convergence.

The first initial-boundary value problem for infinite systems of weakly coupled differential-functional equations of parabolic type was dealt with using the same monotone iterative technique in [2], [3].

The existence and uniqueness of the Cauchy problem for a parabolic equation with functional dependence represented by a Hale type operator and comparison principles for such a parabolic differential-functional initial value problem were considered in [4] and [1], respectively.

$\left({ }^{1}\right)$ That is, every equation contains all unknown functions and derivatives of only one unknown function. 
This paper is organized as follows. In the next section the necessary notations are introduced. We also formulate our assumptions and auxiliary lemmas. In Section 3 we state and prove comparison principles for bounded continuous functions and a theorem on the existence and uniqueness of solutions in that class of functions. The last section contains results analogous to ones from Section 3, but for unbounded continuous functions satisfying a certain growth condition.

2. Notations, definitions and assumptions. Throughout the paper, we use the following notation. The Euclidean norm in $\mathbb{R}^{m}$ is denoted by $|\cdot|$ and the norms in function spaces are denoted by $\|\cdot\|$ with appropriate indices, in particular the supremum norm is denoted by $\|\cdot\|_{0}$.

Let $C B_{S}(X)$ denote the space of functions $w=\left\{w^{i}\right\}_{i \in S}$ such that $w \in$ $C_{S}(X)$ and each $w^{i}$ is bounded on $X$, uniformly in $i$. This space, endowed with the supremum norm

$$
\|w\|_{0}:=\sup \left\{\left|w^{i}(x)\right|: x \in X, i \in S\right\}
$$

is a Banach space. For $w \in C B_{S}(\bar{\Omega})$ and for a fixed $t \geq 0$ we define

$$
\|w\|_{0, t}:=\sup \left\{\left|w^{i}(\tilde{t}, x)\right|:(\widetilde{t}, x) \in \bar{\Omega}, \tilde{t} \leq t, i \in S\right\} .
$$

Let $R>0$. Denote by $D_{R}$ the cylinder $\left\{(t, x): \sum_{j=1}^{m} x_{j}^{2} \leq R^{2}, 0 \leq t \leq T\right\}$. Let $\Gamma_{R}:=\left\{(t, x): \sum_{j=1}^{m} x_{j}^{2}=R^{2}, 0 \leq t \leq T\right\}$ and let $S_{R}^{0}$ stand for the base of $D_{R}$, i.e. the set $\left\{(t, x): \sum_{j=1}^{m} x_{j}^{2} \leq R^{2}, t=0\right\}$.

The homogeneous system, i.e.

$$
\mathcal{F}^{i}\left[u^{i}\right](t, x)=0, \quad i \in S,
$$

is a particular case of (1). Observe that it is a system of independent homogeneous equations. The fundamental solutions $\Gamma^{i}(t, x ; \tau, \xi)$ of the homogeneous equations play an important role in our further considerations.

We now formulate the crucial assumptions concerning the coefficients of the operators $\left\{\mathcal{F}^{i}\right\}_{i \in S}$, which are sufficient for the existence of the fundamental solutions for the homogeneous system. These assumptions will be in force throughout the paper.

We assume that

$(\mathcal{P})$ the operators $\mathcal{F}^{i}, i \in S$, are uniformly parabolic in $\bar{\Omega}$, i.e. there is $\mu>0$ such that

$$
\sum_{j, k=1}^{m} a_{j k}^{i}(t, x) \xi_{j} \xi_{k} \geq \mu \sum_{j=1}^{m} \xi_{j}^{2}
$$

for all $(t, x) \in \bar{\Omega}, \xi=\left(\xi_{1}, \ldots, \xi_{m}\right) \in \mathbb{R}^{m}$, and $i \in S$; 
$(\mathcal{H}) \quad$ the coefficients $a_{j k}^{i}(t, x), b_{j}^{i}(t, x), c^{i}(t, x), i \in S, j, k=1, \ldots, m$, are bounded, continuous functions in $\bar{\Omega}$ such that $a_{j k}^{i}(t, x)=a_{k j}^{i}(t, x)$ and satisfy the following uniform Hölder conditions with exponent $\alpha$ $(0<\alpha \leq 1)$ in $\bar{\Omega}$ with respect to $x$ : there exists $H>0$ such that

$$
\begin{aligned}
\left|a_{j k}^{i}(t, x)-a_{j k}^{i}\left(t, x^{\prime}\right)\right| & \leq H\left|x-x^{\prime}\right|^{\alpha}, \\
\left|b_{j}^{i}(t, x)-b_{j}^{i}\left(t, x^{\prime}\right)\right| & \leq H\left|x-x^{\prime}\right|^{\alpha}, \\
\left|c^{i}(t, x)-c^{i}\left(t, x^{\prime}\right)\right| & \leq H\left|x-x^{\prime}\right|^{\alpha},
\end{aligned}
$$

for all $(t, x),\left(t, x^{\prime}\right) \in \bar{\Omega}, j, k=1, \ldots, m$, and $i \in S$.

Now, let us recall a lemma on the existence of the fundamental solution and its estimate, whose proof can be found in [5] or [6].

Lemma 1. If assumptions $(\mathcal{P})$ and $(\mathcal{H})$ hold then there exist the fundamental solutions $\Gamma^{i}(t, x ; \tau, \xi)$ of the equations $\mathcal{F}^{i}\left[u^{i}\right](t, x)=0, i \in S$, and

$$
\left|\Gamma^{i}(t, x ; \tau, \xi)\right| \leq c(t-\tau)^{-m / 2} \exp \left(-\frac{\mu^{*}|x-\xi|^{2}}{4(t-\tau)}\right), \quad i \in S,
$$

for some $\mu^{*}<\mu$, where $\mu^{*}$ depends on $\mu$ and $H$, whereas $c$ depends on $\mu, \alpha$, $T$ and the character of continuity of $a_{j k}^{i}(t, x)$ in $t$.

Let us notice that from the proof of this lemma and the above assumptions on the coefficients of the operators $\mathcal{F}^{i}, i \in S$, it follows that the constants $c$ and $\mu^{*}$ are independent of $i$.

From the above inequality it follows immediately that

$$
\int_{\mathbb{R}^{m}}\left|\Gamma^{i}(t, x ; \tau, \xi)\right| d \xi \leq C \quad \text { for } i \in S, \quad \text { where } \quad C=c\left(4 \pi / \mu^{*}\right)^{m / 2} .
$$

The notation for constants which appear in Lemma 1 will be valid throughout the paper.

If we strengthen the assumption on the principal coefficients of the operators $\left\{\mathcal{F}^{i}\right\}_{i \in S}$ then we obtain the positivity of the fundamental solution (cf. [6]). Therefore, we will assume, instead of condition $(\mathcal{H})$, the following condition $\left(\mathcal{H}_{t}\right)$ :

$\left(\mathcal{H}_{\boldsymbol{t}}\right)$ the coefficients $a_{j k}^{i}(t, x), b_{j}^{i}(t, x), c^{i}(t, x), i \in S, j, k=1, \ldots, m$, are bounded, continuous functions in $\Omega$ with $a_{j k}^{i}(t, x)=a_{k j}^{i}(t, x)$ and satisfy the following uniform Hölder conditions with exponent $\alpha$ $(0<\alpha \leq 1)$ in $\bar{\Omega}$ : there exists $H>0$ such that

$$
\begin{aligned}
\left|a_{j k}^{i}(t, x)-a_{j k}^{i}\left(t^{\prime}, x^{\prime}\right)\right| & \leq H\left(\left|x-x^{\prime}\right|^{\alpha}+\left|t-t^{\prime}\right|^{\alpha / 2}\right), \\
\left|b_{j}^{i}(t, x)-b_{j}^{i}\left(t, x^{\prime}\right)\right| & \leq H\left|x-x^{\prime}\right|^{\alpha}, \\
\left|c^{i}(t, x)-c^{i}\left(t, x^{\prime}\right)\right| & \leq H\left|x-x^{\prime}\right|^{\alpha},
\end{aligned}
$$

for all $t, t^{\prime} \in[0, T], x, x^{\prime} \in \mathbb{R}^{m}, j, k=1, \ldots, m$ and $i \in S$. 
LEMMA 2. If assumptions $(\mathcal{P})$ and $\left(\mathcal{H}_{\boldsymbol{t}}\right)$ hold then the $\Gamma^{i}(t, x ; \tau, \xi)$ are positive functions.

Using the fundamental solutions we can transform the differential problem (1), (2) into the following associated integral system:

$$
\begin{aligned}
u^{i}(t, x) & =\int_{\mathbb{R}^{m}} \Gamma^{i}(t, x ; 0, \xi) \varphi^{i}(\xi) d \xi \\
& +\int_{0}^{t} \int_{\mathbb{R}^{m}} \Gamma^{i}(t, x ; \tau, \xi) f^{i}(\tau, \xi, u(\tau, \xi), u) d \xi d \tau \quad \text { for } t>0, x \in \mathbb{R}^{m} .
\end{aligned}
$$

In the space $C_{S}(\bar{\Omega})$ the following partial order is introduced: for $z, \widetilde{z} \in$ $C_{S}(\bar{\Omega})$, the inequality $z \leq \widetilde{z}$ means that

$$
z^{i}(t, x) \leq \bar{z}^{i}(t, x) \quad \text { for all }(t, x) \in \bar{\Omega} \text { and } i \in S .
$$

Now, we recall the definitions of subsolutions and supersolutions.

Definition. Functions $v=\left\{v^{i}\right\}_{i \in S}, w=\left\{w^{i}\right\}_{i \in S}$ such that $v^{i}, w^{i} \in$ $C^{1,2}(\bar{\Omega})$ for all $i \in S$ satisfy the system of inequalities

$$
\begin{aligned}
\mathcal{F}^{i}\left[v^{i}\right](t, x) & \leq f^{i}(t, x, v(t, x), v) & & \text { for }(t, x) \in \Omega, i \in S, \\
v(0, x) & \leq \varphi(x) & & \text { for } x \in \mathbb{R}^{m}, \\
\mathcal{F}^{i}\left[w^{i}\right](t, x) & \geq f^{i}(t, x, w(t, x), w) & & \text { for }(t, x) \in \Omega, i \in S, \\
w(0, x) & \geq \varphi(x) & & \text { for } x \in \mathbb{R}^{m} .
\end{aligned}
$$

are called, respectively, a subsolution and a supersolution for problem (1), (2) in $\bar{\Omega}$.

This definition can be extended to the case when $v=\left\{v^{i}\right\}_{i \in S}$ and $w=$ $\left\{w^{i}\right\}_{i \in S}$ are less regular, namely $v, w \in C_{S}(\bar{\Omega})$. Then $v=\left\{v^{i}\right\}_{i \in S}$ and $w=$ $\left\{w^{i}\right\}_{i \in S}$ are called a sub- and supersolution if they satisfy the inequalities $v \leq \mathbf{T}[v]$ and $w \geq \mathbf{T}[w]$, respectively, where $\mathbf{T}=\left\{\mathbf{T}^{i}\right\}_{i \in S}$ is the right hand side of (4), i.e.

$$
\mathbf{T}^{i}[v]=\int_{\mathbb{R}^{m}} \Gamma^{i}(t, x ; 0, \xi) \varphi^{i}(\xi) d \xi+\int_{0}^{t} \int_{\mathbb{R}^{m}} \Gamma^{i}(t, x ; \tau, \xi) f^{i}(\tau, \xi, v(\tau, \xi), v) d \xi d \tau .
$$

In that case one can say that the differential inequalities

$$
\mathcal{F}^{i}\left[v^{i}\right](t, x) \leq f^{i}(t, x, v(t, x), v), \quad i \in S,
$$

are satisfied in the integral sense $(v \leq \mathbf{T}[v])$.

To end this section we recall a technical but useful lemma ([1], [4]), which we use in Section 4. 
Lemma 3. If $0 \leq B<A$ then

$$
\int_{\mathbb{R}^{m}} \exp \left(-A|x-\xi|^{2}+B|\xi|^{2}\right) d \xi=\left(\frac{\pi}{A-B}\right)^{m / 2} \exp \left(\frac{A B}{A-B}|x|^{2}\right) .
$$

This follows from the equality

$$
-A|x-\xi|^{2}+B|\xi|^{2}=\sum_{i=1}^{n} \frac{A B}{A-B} x_{i}^{2}-\left(\sqrt{A-B} \xi_{i}-\frac{A}{\sqrt{A-B}} x_{i}^{2}\right)^{2} .
$$

Now, changing variables as follows: $z_{i}=\sqrt{A-B} \xi_{i}-\frac{A}{\sqrt{A-B}} x_{i}^{2}$, and making use of the fact that $\int_{\mathbb{R}^{m}} \exp \left(-|z|^{2}\right) d z=\pi^{m / 2}$ yields the result.

3. Bounded solutions. We begin this section with a proposition on the continuous dependence of bounded solutions of the Cauchy problem on the right hand sides and initial values. In case the right hand sides satisfy the Lipschitz condition, this guarantees the uniqueness of a solution of problem (1), (2).

Proposition 1. Let $f^{i}=f^{i}(t, x, s, p)$ be Lipschitz continuous in $s$ and in $p$ (uniformly with respect to $i \in S$ ), i.e.

$$
\left|f^{i}(t, x, s, p)-f^{i}(t, x, \widetilde{s}, \widetilde{p})\right| \leq L_{1}\|s-\widetilde{s}\|_{B(S)}+L_{2}\|p-\widetilde{p}\|_{0, t} .
$$

If $v, w \in C_{S}(\bar{\Omega})$ satisfy

$$
\begin{aligned}
& \mathcal{F}^{i}\left[v^{i}\right](t, x)=f^{i}(t, x, v(t, x), v), \\
& \mathcal{F}^{i}\left[w^{i}\right](t, x)=\bar{f}^{i}(t, x, w(t, x), w), \quad i \in S,
\end{aligned}
$$

and there exists a nonnegative constant $M$ independent of $i \in S$ such that

$$
\left|f^{i}(t, x, s, p)-\bar{f}^{i}(t, x, s, p)\right| \leq M
$$

then

$$
\|v-w\|_{0, t} \leq C\|v(0, \cdot)-w(0, \cdot)\|_{0} e^{t C L}+\int_{0}^{t} C M e^{(t-\tau) C L} d \tau
$$

provided $v-w \in C B_{S}(\bar{\Omega})$, where $L=L_{1}+L_{2}$.

Proof. Set $\widetilde{z}(t):=\|z(t)\|_{B(S)}$, where $z(t)=\left\{z^{i}(t)\right\}_{i \in S}$ and

$$
z^{i}(t)=\sup _{x \in \mathbb{R}^{m}, \bar{t} \leq t}\left|v^{i}(\bar{t}, x)-w^{i}(\bar{t}, x)\right|=\left\|v^{i}-w^{i}\right\|_{0, t} .
$$

Since $v, w$ satisfy (5), making use of (6) and of the Lipschitz continuity of $f^{i}$ in $s$ and $p$ we have

$$
\begin{aligned}
v^{i}(t, x)-w^{i}(t, x) & =\int_{\mathbb{R}^{m}} \Gamma^{i}(t, x ; 0, \xi)\left[v^{i}(0, \xi)-w^{i}(0, \xi)\right] d \xi \\
& +\int_{0}^{t} \int_{\mathbb{R}^{m}} \Gamma^{i}(t, x ; \tau, \xi)\left[f^{i}(\tau, \xi, v(\tau, \xi), v)-\bar{f}^{i}(\tau, \xi, w(\tau, \xi), w)\right] d \xi d \tau
\end{aligned}
$$




$$
\begin{aligned}
& \leq C z^{i}(0)+\int_{0}^{t} \int_{\mathbb{R}^{m}} \Gamma^{i}(t, x ; \tau, \xi)\left|f^{i}(\tau, \xi, v(\tau, \xi), v)-f^{i}(\tau, \xi, w(\tau, \xi), v)\right| d \xi d \tau \\
& \quad+\int_{0}^{t} \int_{\mathbb{R}^{m}} \Gamma^{i}(t, x ; \tau, \xi)\left|f^{i}(\tau, \xi, w(\tau, \xi), v)-f^{i}(\tau, \xi, w(\tau, \xi), w)\right| d \xi d \tau \\
& \quad+\int_{0}^{t} \int_{\mathbb{R}^{m}} \Gamma^{i}(t, x ; \tau, \xi)\left|f^{i}(\tau, \xi, w(\tau, \xi), w)-\bar{f}^{i}(\tau, \xi, w(\tau, \xi), w)\right| d \xi d \tau \\
& \leq C z^{i}(0)+\int_{0}^{t} C\left(L_{1}\|v(t, x)-w(t, x)\|_{B(S)}+L_{2}\|v-w\|_{0, \tau}+M\right) d \tau \\
& \leq C z^{i}(0)+\int_{0}^{t} C\left[\left(L_{1}+L_{2}\right)\|z(\tau)\|_{B(S)}+M\right] d \tau, \quad i \in S .
\end{aligned}
$$

Therefore $\widetilde{z}$ satisfies the integral inequality

$$
\widetilde{z}(t) \leq C \widetilde{z}(0)+\int_{0}^{t} C\left[M+\left(L_{1}+L_{2}\right) \widetilde{z}(\tau)\right] d \tau .
$$

Thus, the Gronwall lemma yields

$$
\widetilde{z}(t) \leq C \widetilde{z}(0) e^{t C\left(L_{1}+L_{2}\right)}+\int_{0}^{t} C M e^{(t-\tau) C\left(L_{1}+L_{2}\right)} d \tau .
$$

REMARK 1. In particular, if the right hand sides of (5) are equal and $v(0, x)=w(0, x)$ then Proposition 1 yields the uniqueness.

Now, let us state a result concerning weak partial differential-functional inequalities for an infinite system of parabolic equations in the class of continuous bounded functions. The Gronwall lemma will be our main tool in the proof. This proposition plays a fundamental role in the proof of the main theorem of this section, i.e. Theorem 1.

Proposition 2. Let assumptions $(\mathcal{P})$ and $\left(\mathcal{H}_{t}\right)$ hold. Let $f^{i}=f^{i}(t, x$, $s, p)$ be increasing in $s$ and $p$, and satisfy, uniformly with respect to $i \in S$, the one-sided Lipschitz condition with respect to $s$ and $p$, i.e.

$$
\begin{array}{r}
f^{i}(t, x, s, p)-f^{i}(t, x, \widetilde{s}, p) \leq L_{1}\|s-\widetilde{s}\|_{B(S)}+L_{2}\|p-\widetilde{p}\|_{0, t} \\
\text { for } s \geq \widetilde{s}, p \geq \widetilde{p} .
\end{array}
$$

If $v, w \in C B_{S}(\bar{\Omega})$ satisfy

$$
\begin{aligned}
& \mathcal{F}^{i}\left[v^{i}\right](t, x) \leq f^{i}(t, x, v(t, x), v), \\
& \mathcal{F}^{i}\left[w^{i}\right](t, x) \geq f^{i}(t, x, w(t, x), w), \quad i \in S,
\end{aligned}
$$

then the initial inequality $v(0, x) \leq w(0, x)$ carries over to $\bar{\Omega}$. 
Proof. Set $y(t)=\left\{y^{i}(t)\right\}_{i \in S}$ where $y^{i}(t):=\max \left\{0, z^{i}(t)\right\}$ and

$$
z^{i}(t)=\sup _{x \in \mathbb{R}^{m}, \bar{t} \leq t}\left(v^{i}(\bar{t}, x)-w^{i}(\bar{t}, x)\right) .
$$

It is obvious that $0 \leq y^{i}(t)<\infty$ and $z^{i}(t) \leq y^{i}(t)$ for all $i \in S$. Let $\mathbb{I} \in C B_{S}(\bar{\Omega})$ be the function whose every component is the constant function equal to 1 .

Now, the Lipschitz conditions and the fact that $f^{i}, i \in S$, are increasing in $s$ and $p$ yield

$$
\begin{aligned}
f^{i}(t, x, v(t, x) & , v)-f^{i}(t, x, w(t, x), w) \\
& =f^{i}(t, x,[w+(v-w)](t, x),[w+(v-w)])-f^{i}(t, x, w(t, x), w) \\
& \leq f^{i}(t, x, w(t, x)+y(t) \mathbb{I}(t, x), w+y \mathbb{I})-f^{i}(t, x, w(t, x), w) \\
& \leq L_{1}\|y(t)\|_{B(S)}+L_{2}\|y \mathbb{I}\|_{0, t}=\left(L_{1}+L_{2}\right)\|y(t)\|_{B(S)} .
\end{aligned}
$$

Since $v, w$ satisfy (7), for all $i \in S$ we have

$$
\begin{aligned}
v^{i}(t, x)-w^{i}(t, x) & =\int_{\mathbb{R}^{m}} \Gamma^{i}(t, x ; 0, \xi)\left[v^{i}(0, \xi)-w^{i}(0, \xi)\right] d \xi \\
+\int_{0}^{t} \int_{\mathbb{R}^{m}} \Gamma^{i}(t, x ; \tau, \xi)\left[f^{i}(\tau, \xi, v(\tau, \xi), v)-f^{i}(\tau, \xi, w(\tau, \xi), w)\right] d \xi d \tau & \\
& \leq \int_{0}^{t} C\left(L_{1}+L_{2}\right)\|y(\tau)\|_{B(S)} d \tau .
\end{aligned}
$$

Thus, the function $\widetilde{y}(t)=\|y(t)\|_{B(S)}$ satisfies

$$
\widetilde{y}(t) \leq \int_{0}^{t} C\left(L_{1}+L_{2}\right) \widetilde{y}(\tau) d \tau .
$$

Now the Gronwall lemma yields $\widetilde{y}(t) \equiv 0$, which means that $v(t, x) \leq w(t, x)$ in $\bar{\Omega}$.

As a direct consequence of Proposition 2 we get the following corollary.

Corollary 1. Let $u \in C B_{S}(\bar{\Omega})$ and suppose that

$$
\begin{aligned}
\mathcal{F}^{i}\left[u^{i}\right](t, x) \leq 0 & \text { for }(t, x) \in \Omega, \quad i \in S, \\
u(0, x) \leq 0 & \text { for } x \in \mathbb{R}^{m} .
\end{aligned}
$$

Then $u(t, x) \leq 0$ for $(t, x) \in \bar{\Omega}$.

REMARK 2. If $v$ and $w$ are a subsolution and a supersolution for problem (1), (2) in $\bar{\Omega}$, respectively, and $u$ is any solution of this problem, then Proposition 2 yields

$$
v(t, x) \leq u(t, x) \leq w(t, x) \quad \text { for }(t, x) \in \bar{\Omega} .
$$


Below in Theorem 1 we construct certain sequences of successive approximations as solutions of some linear systems of differential equations. Let us stress that Proposition 2 ensures the uniform convergence of these sequences to the required solution of problem (1), (2).

Before formulating the theorem we introduce the following notation.

For every sufficiently smooth function $\beta$, let $\gamma=\mathcal{P}[\beta]$ be the unique solution of the initial value problem

$$
\begin{aligned}
\mathcal{F}^{i}\left[\gamma^{i}\right](t, x) & =f^{i}(t, x, \beta(t, x), \beta), \quad i \in S, \\
\gamma(0, x) & =\varphi(x) \quad \text { for } x \in \mathbb{R}^{m} .
\end{aligned}
$$

Now, let us formulate the following assumptions.

Assumptions 1. All components $f^{i}(t, x, s, p)$ of $f=\left\{f^{i}\right\}_{i \in S}$ are

$\left(C_{f}\right)$ continuous in $\bar{\Omega} \times B(S) \times C B_{S}(\bar{\Omega})$;

$\left(B_{f}\right)$ uniformly bounded in $\bar{\Omega} \times B(S) \times C B_{S}(\bar{\Omega})$;

$\left(I_{f}\right)$ increasing with respect to $s$ and $p$;

$\left(H_{f}\right)$ locally Hölder continuous with respect to $x$ uniformly in $t$;

$\left(L_{f}\right)$ Lipschitz continuous with respect to $s$ and $p$ :

$$
\begin{aligned}
& \left|f^{i}(t, x, s, p)-f^{i}(t, x, \widetilde{s}, \widetilde{p})\right| \leq L_{1}\|s-\widetilde{s}\|_{B(S)}+L_{2}\|p-\widetilde{p}\|_{0, t} \\
& \qquad \text { for }(t, x) \in \Omega, s, \widetilde{s} \in B(S), p, \widetilde{p} \in C_{S}(\bar{\Omega}) .
\end{aligned}
$$

REMARK 3. Since the functions $f^{i}=f^{i}(t, x, s, p), i \in S$, are increasing in $s$ and $p$ and $\Gamma^{i}(t, x ; \tau, \xi)$ is a positive function the operator $\mathcal{P}$ is increasing.

Assumption 2. There exists at least one pair $v_{0}=v_{0}(t, x), w_{0}=$ $w_{0}(t, x) \in C B_{S}(\bar{\Omega})$ of a subsolution and a supersolution of problem (1), (2) in $\bar{\Omega}$ which are Hölder continuous in $x$ uniformly with respect to $t$.

Now, we state and prove the theorem on the existence and uniqueness of solution of problem (1), (2) obtained by a simple iterative method, i.e. starting from a subsolution $v_{0}$ and a supersolution $w_{0}$ we define $v_{n}:=\mathcal{P}\left[v_{n-1}\right]$, $w_{n}:=\mathcal{P}\left[w_{n-1}\right], n=1,2, \ldots$ Thus, at each step we have an infinite system of linear equations. The sequence of successive approximations converges to the desired solution with power speed.

THEOREM 1. Let conditions $(\mathcal{P})$ and $\left(\mathcal{H}_{\boldsymbol{t}}\right)$ hold. Suppose that every component $\varphi^{i}$ of the initial data $\varphi=\left\{\varphi^{i}\right\}_{i \in S}$ is a bounded continuous function on $\mathbb{R}^{m}$. Moreover, let Assumptions 1 and 2 hold. Consider the following infinite system of linear equations:

$$
\begin{aligned}
& \mathcal{F}^{i}\left[v_{n}^{i}\right](t, x)=f^{i}\left(t, x, v_{n-1}(t, x), v_{n-1}\right), \\
& \mathcal{F}^{i}\left[w_{n}^{i}\right](t, x)=f^{i}\left(t, x, w_{n-1}(t, x), w_{n-1}\right),
\end{aligned}
$$

for $(t, x) \in \Omega, i \in S, n=1,2, \ldots$ with the initial condition (2), and let $N_{0}=\left\|w_{0}-v_{0}\right\|_{0}<\infty$. Then 
(i) there exist unique classical bounded solutions $v_{n}$ and $w_{n}, n=1,2, \ldots$, of systems (8) and (9) with the initial condition (2) in $\bar{\Omega}$;

(ii) $v_{n}$ and $w_{n}, n=1,2, \ldots$, are respectively subsolutions and supersolutions for problem (1), (2) in $\bar{\Omega}$;

(iii) we have

$$
\begin{aligned}
v_{0}(t, x) \leq \cdots \leq v_{n}(t, x) & \leq v_{n+1}(t, x) \leq \cdots \\
& \leq w_{n+1}(t, x) \leq w_{n}(t, x) \leq \cdots \leq w_{0}(t, x)
\end{aligned}
$$

for $(t, x) \in \bar{\Omega}, n=1,2 \ldots$

(iv) $\lim _{n \rightarrow \infty}\left[w_{n}^{i}(t, x)-v_{n}^{i}(t, x)\right]=0$ uniformly in $\bar{\Omega}, i \in S$;

(v) $u(t, x)=\lim _{n \rightarrow \infty} v_{n}(t, x)$ is a unique classical bounded solution of problem (1), (2) in $\bar{\Omega}$.

Proof. (i) Starting from $v_{0}$ and $w_{0}$ we define $v_{1}, w_{1}$ as solutions of (8), (9) with the initial condition (2), i.e. $v_{1}=\mathcal{P}\left[v_{0}\right], w_{1}=\mathcal{P}\left[w_{0}\right]$. Observe that the systems in question have the following property: the $i$ th equation depends on the $i$ th unknown function only, therefore since $v_{0}, w_{0}$ satisfy Assumption 2, the classical theorems on the existence and uniqueness of solution of linear parabolic Cauchy problems (cf. [5] or [6]) assert that there exist unique solutions $v_{1}, w_{1} \in C B_{S}(\bar{\Omega})$ of the above problems and $v_{1}$ and $w_{1}$ are Hölder continuous with respect to $x$ uniformly in $t$ (cf. [5]).

Next, we define by induction $\left\{v_{n}\right\},\left\{w_{n}\right\}$ as solutions of (8), (9) with the initial condition (2), i.e. $v_{n}=\mathcal{P}\left[v_{n-1}\right], w_{n}=\mathcal{P}\left[w_{n-1}\right]$.

The preceding reasoning shows that $v_{n}, w_{n}$ exist and are uniquely defined. Moreover, for each $i \in S, n=1,2, \ldots, v_{n}^{i}, w_{n}^{i}$ are bounded, belong to $C^{1,2}(\bar{\Omega})$ and are Hölder continuous in $x$ uniformly in $t$.

(ii) We now show, by induction, that the functions $v_{n}$ are subsolutions. $v_{0}$ is a subsolution by Assumption 2. Suppose $v_{n}$ is a subsolution of (1), (2) in $\bar{\Omega}$, i.e.

$$
\begin{aligned}
\mathcal{F}^{i}\left[v_{n}^{i}\right](t, x) & \leq f^{i}\left(t, x, v_{n}(t, x), v_{n}\right) & & \text { for }(t, x) \in \Omega, i \in S, \\
v_{n}(0, x) & \leq \varphi(x) & & \text { for } x \in \mathbb{R}^{m} .
\end{aligned}
$$

From the definition of the operator $\mathcal{P}$ it follows that

$$
\begin{aligned}
\mathcal{F}^{i}\left[v_{n+1}^{i}\right](t, x) & =f^{i}\left(t, x, v_{n}(t, x), v_{n}\right) & & \text { for }(t, x) \in \Omega, i \in S, \\
v_{n+1}(0, x) & =\varphi(x) & & \text { for } x \in \mathbb{R}^{m} .
\end{aligned}
$$

Thus, Corollary 1 yields

$$
\left[v_{n}-v_{n+1}\right](t, x) \leq 0 \quad \text { for }(t, x) \in \bar{\Omega}
$$

i.e.

$$
v_{n}(t, x) \leq \mathcal{P}\left[v_{n}\right](t, x) \quad \text { for }(t, x) \in \bar{\Omega} .
$$

Now, the monotonicity condition $\left(I_{f}\right)$ implies 


$$
\begin{aligned}
\mathcal{F}^{i}\left[v_{n+1}^{i}\right](t, x)- & f^{i}\left(t, x, v_{n+1}(t, x), v_{n+1}\right) \\
& =f^{i}\left(t, x, v_{n}(t, x), v_{n}\right)-f^{i}\left(t, x, \mathcal{P}\left[v_{n}\right](t, x), \mathcal{P}\left[v_{n}\right]\right) \leq 0
\end{aligned}
$$

for all $i \in S,(t, x) \in \bar{\Omega}$. We conclude that $v_{n+1}$ is a subsolution as well. The proof that the $w_{n}$ are supersolutions is similar.

(iii) The monotonicity of the sequences $\left\{v_{n}\right\},\left\{w_{n}\right\}$ is a consequence of the fact that $v_{n}, w_{n}$ are subsolutions and supersolutions, respectively, whereas the inequality $v_{n} \leq w_{n}$ follows from the monotonicity of the operator $\mathcal{P}$ and the fact that $v_{0} \leq w_{0}$.

(iv) We show by induction that $m_{n}^{i}(t, x):=w_{n}^{i}(t, x)-v_{n}^{i}(t, x) \geq 0$ is estimated as follows:

$$
m_{n}^{i}(t, x) \leq N_{0} \frac{\left[\left(L_{1}+L_{2}\right) t\right]^{n}}{n !}, \quad n=0,1 \ldots, \text { for }(t, x) \in \bar{\Omega}, i \in S .
$$

The inequality for $m_{0}^{i}$ is obvious. Suppose it holds for $m_{n}^{i}$. Condition $\left(L_{f}\right)$ yields

$$
\begin{aligned}
\mathcal{F}^{i}\left[m_{n+1}^{i}\right](t, x) & =f^{i}\left(t, x, w_{n}(t, x), w_{n}\right)-f^{i}\left(t, x, v_{n}(t, x), v_{n}\right) \\
& \leq L_{1}\left\|m_{n}(t, x)\right\|_{B(S)}+L_{2}\left\|m_{n}\right\|_{0, t} .
\end{aligned}
$$

By the definitions of $\|\cdot\|_{0, t}$ and $\|\cdot\|_{B(S)}$ and the induction assumption both $\left\|m_{n}(t, x)\right\|_{B(S)}$ and $\left\|m_{n}\right\|_{0, t}$ are estimated by $N_{0}\left[\left(L_{1}+L_{2}\right) t\right]^{n} / n !$. Thus, finally,

$$
\mathcal{F}^{i}\left[m_{n+1}^{i}\right](t, x) \leq N_{0} \frac{\left(L_{1}+L_{2}\right)^{n+1} t^{n}}{n !} \quad \text { in } \Omega
$$

and $m_{n+1}^{i}(0, x)=0$ for $x \in \mathbb{R}^{m}, i \in S$.

In order to apply the theorem on differential inequalities, consider the comparison system

$$
\mathcal{F}^{i}\left[M_{n+1}^{i}\right](t, x)=N_{0} \frac{\left(L_{1}+L_{2}\right)^{n+1} t^{n}}{n !} \quad \text { for }(t, x) \in \Omega, i \in S,
$$

with the initial condition $M_{n+1}^{i}(0, x) \geq 0$ for $x \in \mathbb{R}^{m}, i \in S$.

The functions $M_{n+1}^{i}(t, x)=N_{0}\left[\left(L_{1}+L_{2}\right) t\right]^{n+1} /(n+1)$ ! are solutions of the comparison problem, therefore, owing to Proposition 2, we get

$$
m_{n+1}^{i}(t, x) \leq M_{n+1}^{i}(t, x)=N_{0} \frac{\left[\left(L_{1}+L_{2}\right) t\right]^{n+1}}{(n+1) !} \quad \text { for }(t, x) \in \bar{\Omega}, i \in S,
$$

so, the induction step is proved. As a direct consequence of (10) we obtain

$$
\lim _{n \rightarrow \infty}\left[w_{n}^{i}(t, x)-v_{n}^{i}(t, x)\right]=0 \quad \text { uniformly in } \bar{\Omega} .
$$

(v) First, notice that, since $\left\{v_{n}\right\}$ and $\left\{w_{n}\right\}$ are bounded and monotone sequences of continuous functions and (11) holds, there exist continuous bounded functions $u^{i}=u^{i}(t, x)$ such that

$$
\lim _{n \rightarrow \infty} v_{n}^{i}(t, x)=u^{i}(t, x), \quad \lim _{n \rightarrow \infty} w_{n}^{i}(t, x)=u^{i}(t, x)
$$


uniformly in $\bar{\Omega}$ for all $i \in S$ and the function $u=\left\{u^{i}\right\}_{i \in S}$ satisfies the initial condition (2).

Now, we prove that $u$ satisfies (1). It is enough to show that $u$ fulfills (1) in any compact set contained in $\Omega$.

Consequently, we only need to prove it in $D_{R}$ for any $R>0$.

From $\left(I_{f}\right)$ and (iii) it follows that $f^{i}\left(t, x, v_{n-1}(t, x), v_{n-1}\right)$ are uniformly bounded in $D_{R}$ (with respect to $n$ ), therefore the solution $v_{n}(t, x)$ of the linear system

$$
\mathcal{F}^{i}\left[v_{n}^{i}\right](t, x)=f^{i}\left(t, x, v_{n-1}(t, x), v_{n-1}\right), \quad i \in S,
$$

with a suitable initial condition is Hölder continuous with exponent $\alpha$ with respect to $x$ uniformly in $t$, with a constant independent of $n$ (cf. [5]). Hence, $u(t, x)$ also satisfies the Hölder condition with respect to $x$ uniformly in $t$.

Now, consider the system

$$
\mathcal{F}^{i}\left[z^{i}\right](t, x)=f^{i}(t, x, u(t, x), u) \quad \text { for }(t, x) \in D_{R}, i \in S,
$$

with the conditions

$$
\begin{array}{ll}
z(t, x)=u(t, x) & \text { on } \Gamma_{R}, \\
z(0, x)=\varphi(x) & \text { on } S_{R}^{0} .
\end{array}
$$

As $u(t, x)$ is Hölder continuous with respect to $x$ and conditions $\left(H_{f}\right)$ and $\left(L_{f}\right)$ hold, the right hand sides of this system are continuous in $D_{R}$ and locally Hölder continuous with respect to $x$.

Thus, the classical existence and uniqueness theorems for linear parabolic initial-boundary valued problems (cf. [6]) imply that there exists a unique classical solution $z(t, x)$ of the problem (14), (15), (16) in $\bar{D}_{R}$.

On the other hand, from (12) and $\left(L_{f}\right)$ it follows that

$\lim _{n \rightarrow \infty} f^{i}\left(t, x, v_{n-1}(t, x), v_{n-1}\right)=f^{i}(t, x, u(t, x), u) \quad$ uniformly in $\bar{D}_{R}$.

Moreover, the boundary values $v_{n}(t, x)$ converge uniformly to $u(t, x)$ on $\Gamma_{R}$ and the initial values are equal, so applying to systems (13) and (14) the theorem on the continuous dependence of the solution on the right hand sides and initial-boundary values (cf. [11]) we conclude that

$$
\lim _{n \rightarrow \infty} v_{n}^{i}(t, x)=z^{i}(t, x) \quad \text { uniformly in } \bar{D}_{R} \text {. }
$$

Thus $z^{i}(t, x)=u^{i}(t, x)$ in $\bar{D}_{R}$ for all $i \in S$, for arbitrary $R>0$, which means $z(t, x)=u(t, x)$ for all $(t, x) \in \bar{\Omega}$, i.e. $u(t, x)$ is a classical bounded solution of problem (1), (2).

The uniqueness of the solution is a direct consequence of Remark 1. Theorem 1 is proved.

4. Unbounded solutions. This section contains a result (Proposition 3) which is crucial in the proof of our theorem on the existence of unbounded 
solutions (Theorem 2). It yields weak partial differential inequalities for functions which behave like $\left|u^{i}(t, x)\right| \leq D \exp \left(d|x|^{2}\right)$. The result is obtained upon assuming the right hand sides to satisfy the Lipschitz condition with respect to $s$ and a weighted Lipschitz condition with respect to $p$.

We denote by $C^{+}$the space of all positive, real-valued, continuous and nondecreasing functions defined on the set $[0, T]$. For $w \in C_{S}(\bar{\Omega})$ we define the following weighted norms depending on $\phi \in C^{+}$:

$$
\begin{aligned}
\|w\|_{2, \phi} & :=\sup _{i \in S} \sup _{(t, x) \in \bar{\Omega}} \frac{\left|w^{i}(t, x)\right|}{[\phi(t)]^{m / 2} \exp \left(\phi(t)|x|^{2}\right)}, \\
\|w\|_{2, \phi, t} & :=\sup _{i \in S} \sup _{x \in \mathbb{R}^{m}, \bar{t} \leq t} \frac{\left|w^{i}(\bar{t}, x)\right|}{[\phi(\bar{t})]^{m / 2} \exp \left(\phi(\bar{t})|x|^{2}\right)} .
\end{aligned}
$$

Let $E_{S}^{2, \phi}$ for $\phi \in C^{+}$be the space of all functions $w \in C_{S}(\bar{\Omega})$ such that

$$
\exists D \geq 0 \forall(t, x) \in \bar{\Omega} \forall i \in S \quad\left|w^{i}(t, x)\right| \leq D \exp \left(\phi(t)|x|^{2}\right) .
$$

Obviously, $E_{S}^{2, \phi}$ endowed with the norm $\|\cdot\|_{2, \phi}$ is a Banach space.

Now, we state and prove the proposition mentioned above.

Proposition 3. Let assumptions $(\mathcal{P})$ and $\left(\mathcal{H}_{\boldsymbol{t}}\right)$ hold. Let $\phi \in C^{+}$satisfy

$$
\frac{\mu^{*} \phi(\tau)}{\mu^{*}-4 \phi(\tau)(t-\tau)} \leq \phi(t) \quad \text { for } 0 \leq \tau \leq t \leq T,
$$

where $\mu^{*}$ is the constant which appeared in Lemma 1 . Let $f^{i}=f^{i}(t, x, s, p)$ be increasing in $s$ and $p$ and satisfy the following one-sided Lipschitz condition uniformly with respect to $i \in S$ :

$$
\begin{aligned}
f^{i}(t, x, s, p)- & f^{i}(t, x, \widetilde{s}, \widetilde{p}) \\
\leq & L_{1}\|s-\widetilde{s}\|_{B(S)}+L_{2}\|p-\widetilde{p}\|_{2, \phi, t}[\phi(t)]^{m / 2} \exp \left(\phi(t)|x|^{2}\right) \\
& \quad \text { for }(t, x) \in \bar{\Omega}, s \geq \widetilde{s}, p \geq \widetilde{p} .
\end{aligned}
$$

If $v, w \in E_{S}^{2, \phi}$ satisfy the system of inequalities

$$
\begin{aligned}
& \mathcal{F}^{i}\left[v^{i}\right](t, x) \leq f^{i}(t, x, v(t, x), v), \\
& \mathcal{F}^{i}\left[w^{i}\right](t, x) \geq f^{i}(t, x, w(t, x), w), \quad i \in S,
\end{aligned}
$$

then the initial inequality $v(0, x) \leq w(0, x)$ carries over to the whole domain.

Proof. As in the proof of Proposition 2 we define $y(t)=\left\{y^{i}(t)\right\}_{i \in S}$, where $y^{i}(t):=\max \left\{0, z^{i}(t)\right\}, z^{i}(t)=\sup _{x \in \mathbb{R}^{m}, \bar{t} \leq t}\left(v^{i}(\bar{t}, x)-w^{i}(\bar{t}, x)\right)$. It is obvious that $0 \leq y^{i}(t)<\infty$ and $z^{i}(t) \leq y^{i}(t)$ for all $i \in S$. Let $\mathbb{I} \in E_{S}^{2, \phi}$ be the constant function equal to 1 .

We notice that the definitions of $\|\cdot\|_{2, \phi, t}$ and $y(t)$ and the monotonicity of $\phi$ yield

$$
\|y(t)\|_{B(S)} \leq\|y\|_{2, \phi, t}[\phi(t)]^{m / 2} \exp \left(\phi(t)|x|^{2}\right)
$$


Combining the Lipschitz condition with the fact that $f^{i}, i \in S$, are increasing in $s$ and $p$ we obtain

$$
\begin{aligned}
& f^{i}(t, x, v(t, x), v)-f^{i}(t, x, w(t, x), w) \\
& =f^{i}(t, x,[w+(v-w)](t, x),[w+(v-w)])-f^{i}(t, x, w(t, x), w) \\
& \leq f^{i}(t, x, w(t, x)+y(t) \mathbb{I}(t, x), w+y \mathbb{I})-f^{i}(t, x, w(t, x), w) \\
& \leq L_{1}\|y(t)\|_{B(S)}+L_{2}\|y\|_{2, \phi, t}[\phi(t)]^{m / 2} \exp \left(\phi(t)|x|^{2}\right) \\
& \leq\left(L_{1}+L_{2}\right)\|y\|_{2, \phi, t}[\phi(t)]^{m / 2} \exp \left(\phi(t)|x|^{2}\right) .
\end{aligned}
$$

Since $v, w$ satisfy (17), making use of (18) and Lemma 1 we have, for all $i \in S$,

$$
\begin{gathered}
\left|v^{i}(t, x)-w^{i}(t, x)\right| \leq \int_{\mathbb{R}^{m}} \Gamma^{i}(t, x ; 0, \xi)\left|v^{i}(0, \xi)-w^{i}(0, \xi)\right| d \xi \\
\quad+\int_{0 \mathbb{R}^{m}} \Gamma^{i}(t, x ; \tau, \xi)\left|f^{i}(\tau, \xi, v(\tau, \xi), v)-f^{i}(\tau, \xi, w(\tau, \xi), w)\right| d \xi d \tau \\
\leq \int_{0 \mathbb{R}^{m}}^{t}\left(L_{1}+L_{2}\right)\|v-w\|_{2, \phi, \tau} \Gamma^{i}(t, x ; \tau, \xi)[\phi(\tau)]^{m / 2} \exp \left(\phi(\tau)|\xi|^{2}\right) d \xi d \tau \\
\leq\left(L_{1}+L_{2}\right) \int_{0}^{t}\|v-w\|_{2, \phi, \tau}[\phi(\tau)]^{m / 2} \int_{\mathbb{R}^{m}} c(t-\tau)^{-m / 2} \\
\times \exp \left(-\frac{\mu^{*}|x-\xi|^{2}}{4(t-\tau)}\right) \exp \left(\phi(\tau)|\xi|^{2}\right) d \xi d \tau .
\end{gathered}
$$

Now, Lemma 3 shows that

$$
\begin{aligned}
& \left|v^{i}(t, x)-w^{i}(t, x)\right| \\
& \begin{aligned}
\leq c\left(L_{1}+L_{2}\right) \int_{0}^{t}\|v-w\|_{2, \phi, \tau}[\phi(\tau)]^{m / 2}(t-\tau)^{-m / 2}\left(\frac{4 \pi(t-\tau)}{\mu^{*}-4 \phi(\tau)(t-\tau)}\right)^{m / 2} \\
\quad \times \exp \left(\frac{\mu^{*} \phi(\tau)|x|^{2}}{\mu^{*}-4 \phi(\tau)(t-\tau)}\right) d \tau \\
\leq c\left(L_{1}+L_{2}\right) \int_{0}^{t}\|v-w\|_{2, \phi, \tau}\left(\frac{4 \pi \phi(\tau)}{\mu^{*}-4 \phi(\tau)(t-\tau)}\right)^{m / 2} \\
\times \exp \left(\frac{\mu^{*} \phi(\tau)|x|^{2}}{\mu^{*}-4 \phi(\tau)(t-\tau)}\right) d \tau .
\end{aligned}
\end{aligned}
$$

By the assumption on $\phi$ we have

$$
\begin{aligned}
& \left|v^{i}(t, x)-w^{i}(t, x)\right| \\
& \quad \leq c\left(L_{1}+L_{2}\right)\left(\frac{4 \pi}{\mu^{*}}\right)^{m / 2}[\phi(t)]^{m / 2} \exp \left(\phi(t)|x|^{2}\right) \int_{0}^{t}\|v-w\|_{2, \phi, \tau} d \tau .
\end{aligned}
$$


Thus,

$$
\|v-w\|_{2, \phi, t} \leq \int_{0}^{t}\left(L_{1}+L_{2}\right)\|v-w\|_{2, \phi, \tau} d \tau .
$$

Now, the Gronwall lemma yields $\|v-w\|_{2, \phi, t} \equiv 0$, so $v(t, x) \leq w(t, x)$ in $\bar{\Omega}$, which completes the proof.

As a direct consequence of Proposition 3 one gets the following corollary.

Corollary 2. Let $u \in E_{S}^{2, \phi}$ and assume that

$$
\begin{aligned}
\mathcal{F}^{i}\left[u^{i}\right](t, x) \leq 0 & \text { for }(t, x) \in \Omega, i \in S, \\
u(0, x) \leq 0 & \text { for } x \in \mathbb{R}^{m} .
\end{aligned}
$$

Then $u(t, x) \leq 0$ for $(t, x) \in \bar{\Omega}$.

REMARK 4. In particular, Proposition 3 shows that there exists at most one solution of problem (1), (2) (in the integral sense) satisfying the growth condition $\left|u^{i}(t, x)\right| \leq D \exp \left(\phi(t)|x|^{2}\right)$ for $(t, x) \in \bar{\Omega}$.

Now, we state a result similar to Theorem 1 , but concerning functions which behave like $\left|u^{i}(t, x)\right| \leq D \exp \left(\phi(t)|x|^{2}\right)$. But first, let us formulate appropriate assumptions.

Assumptions 3. All components $f^{i}(t, x, s, p)$ of $f=\left\{f^{i}\right\}_{i \in S}$ are

$\left(C_{f}\right)$ continuous in $\bar{\Omega} \times B(S) \times E_{S}^{2, \phi}$;

$\left(B_{f}\right)$ exponentially bounded:

$$
\exists M_{0} \geq 0 \forall i \in S \forall(t, x) \in \bar{\Omega} \quad\left|f^{i}(t, x, 0,0)\right| \leq M_{0} \exp \left(\phi(t)|x|^{2}\right) ;
$$

$\left(I_{f}\right)$ increasing with respect to $s$ and $p$;

$\left(H_{f}\right)$ locally Hölder continuous with respect to $x$ uniformly in $t$;

$\left(L_{f}\right)$ weighted Lipschitz continuous in the following sense:

$$
\begin{aligned}
& \qquad \begin{array}{l}
\left|f^{i}(t, x, s, p)-f^{i}(t, x, \widetilde{s}, \widetilde{p})\right| \\
\qquad L_{1}\|s-\widetilde{s}\|_{B(S)}+L_{2}\|p-\widetilde{p}\|_{2, \phi, t}[\phi(t)]^{m / 2} \exp \left(\phi(t)|x|^{2}\right) \\
\text { for }(t, x) \in \Omega, s, \widetilde{s} \in B(S), p, \widetilde{p} \in C_{S}(\bar{\Omega}) .
\end{array}
\end{aligned}
$$

Assumption 4. There exists at least one pair $v_{0}=v_{0}(t, x), w_{0}=w_{0}(t, x)$ $\in E_{S}^{2, \phi}$ of a subsolution and a supersolution of problem (1), (2) in $\bar{\Omega}$ which are Hölder continuous in $x$ uniformly with respect to $t$.

TheOREM 2. Let assumptions $(\mathcal{P})$ and $\left(\mathcal{H}_{\boldsymbol{t}}\right)$ hold. Let $\phi \in C^{+}$satisfy

$$
\frac{\mu^{*} \phi(\tau)}{\mu^{*}-4 \phi(\tau)(t-\tau)} \leq \phi(t) \quad \text { for } 0 \leq \tau \leq t \leq T,
$$

where $\mu^{*}$ is the constant which appeared in Lemma 1. Let $f=\left\{f^{i}\right\}_{i \in S}$ satisfy Assumptions 3. Moreover, let Assumption 4 hold and let all the components 
of the initial data $\varphi=\left\{\varphi^{i}\right\}_{i \in S}$ be such that $\left|\varphi^{i}(x)\right| \leq \bar{K} \exp \left(\phi(0)|x|^{2}\right)$ for all $x \in \mathbb{R}^{m}$.

Consider the following infinite system of linear equations:

$$
\begin{aligned}
\mathcal{F}^{i}\left[v_{n}^{i}\right](t, x) & =f^{i}\left(t, x, v_{n-1}(t, x), v_{n-1}\right), \\
\mathcal{F}^{i}\left[w_{n}^{i}\right](t, x) & =f^{i}\left(t, x, w_{n-1}(t, x), w_{n-1}\right),
\end{aligned}
$$

for $(t, x) \in \Omega, i \in S, n=1,2, \ldots$ with the initial condition (2), and let $N_{0}=\left\|w_{0}-v_{0}\right\|_{2, \phi}<\infty$. Then

(i) there exist unique classical solutions $v_{n} \in E_{S}^{2, \phi}$ and $w_{n} \in E_{S}^{2, \phi}, n=$ $1,2 \ldots$, of systems (19) and (20) with the initial condition $(2)$ in $\bar{\Omega}$;

(ii) $v_{n}$ and $w_{n}, n=1,2, \ldots$, are respectively subsolutions and supersolutions for problem (1), (2) in $\bar{\Omega}$;

(iii) we have

$$
\begin{aligned}
v_{0}(t, x) \leq \cdots \leq v_{n}(t, x) & \leq v_{n+1}(t, x) \leq \cdots \\
& \leq w_{n+1}(t, x) \leq w_{n}(t, x) \leq \cdots \leq w_{0}(t, x)
\end{aligned}
$$

for $(t, x) \in \bar{\Omega}, n=1,2, \ldots$;

(iv) $u(t, x)=\lim _{n \rightarrow \infty} v_{n}(t, x)$ is a unique classical solution of problem (1), (2) in $\bar{\Omega}$ satisfying the condition $\left|u^{i}(t, x)\right| \leq D \exp \left(\phi(t)|x|^{2}\right)$ for $(t, x) \in \bar{\Omega}$.

Proof. (i) As in the proof of Theorem 1, starting from $v_{0}$ and $w_{0}$, we define by induction the sequences $\left\{v_{n}\right\},\left\{w_{n}\right\}$ as solutions of (19), (20) with the initial condition $(2)$ in $\bar{\Omega}$, i.e.

$$
v_{n}=\mathcal{P}\left[v_{n-1}\right], \quad w_{n}=\mathcal{P}\left[v_{n-1}\right] \quad \text { for } n=1,2, \ldots
$$

Here too, the $i$ th equation depends on the $i$ th unknown function only and Assumption 4 holds, therefore the classical theorems on the existence and uniqueness of solution for linear Cauchy problems assert that there exist unique classical solutions $v_{n}, w_{n}$ in $E_{S}^{2, \phi}$ of problems (19), (2) and (20), (2), respectively (cf. [6]).

The proofs of steps (ii)-(iii) are analogous to those in Theorem 1, with Corollary 1 replaced by Corollary 2 and on noticing that the inequalities (iii) guarantee that $u$ satisfies the desired growth condition.

(iv) First, we show that $u(t, x)=\lim _{n \rightarrow \infty} v_{n}(t, x)$ is continuous. To this end we show by induction that $m_{n}^{i}(t, x):=w_{n}^{i}(t, x)-v_{n}^{i}(t, x) \geq 0$ satisfies

$$
\begin{aligned}
& m_{n}^{i}(t, x) \leq N_{0} \frac{\left[\left(L_{1}+L_{2}\right) t\right]^{n}}{n !}[\phi(t)]^{m / 2} \exp \left(\phi(t)|x|^{2}\right), \\
& \quad \text { for }(t, x) \in \bar{\Omega}, n=0,1, \ldots, i \in S .
\end{aligned}
$$

The inequality for $m_{0}^{i}$ is obvious. Suppose it holds for $m_{n}^{i}$. Similarly to the proof of Theorem 1 , the $\left(L_{f}\right)$ condition yields 


$$
\begin{aligned}
\mathcal{F}^{i}\left[m_{n+1}^{i}\right](t, x) & =f^{i}\left(t, x, w_{n}(t, x), w_{n}\right)-f^{i}\left(t, x, v_{n}(t, x), v_{n}\right) \\
& \leq L_{1}\left\|m_{n}(t, x)\right\|_{B(S)}+L_{2}\left\|m_{n}\right\|_{2, \phi, t}[\phi(t)]^{m / 2} \exp \left(\phi(t)|x|^{2}\right) \\
& \leq\left(L_{1}+L_{2}\right)\left\|m_{n}\right\|_{2, \phi, t}[\phi(t)]^{m / 2} \exp \left(\phi(t)|x|^{2}\right) .
\end{aligned}
$$

By the definitions of $\|\cdot\|_{2, \phi, t}$ and the induction assumption, $\left\|m_{n}\right\|_{2, \phi, t} \leq$ $N_{0}\left[\left(L_{1}+L_{2}\right) t\right]^{n} / n !$.

Thus, finally,

$$
\mathcal{F}^{i}\left[m_{n+1}^{i}\right](t, x) \leq N_{0} \frac{\left(L_{1}+L_{2}\right)^{n+1} t^{n}}{n !}[\phi(t)]^{m / 2} \exp \left(\phi(t)|x|^{2}\right) \quad \text { in } \Omega
$$

and $m_{n+1}^{i}(0, x)=0$ for $x \in \mathbb{R}^{m}, i \in S$.

In order to apply the theorem on differential inequalities, consider the comparison system

$$
\mathcal{F}^{i}\left[M_{n+1}^{i}\right](t, x)=N_{0} \frac{\left(L_{1}+L_{2}\right)^{n+1} t^{n}}{n !}[\phi(t)]^{m / 2} \exp \left(\phi(t)|x|^{2}\right)
$$

for $(t, x) \in \bar{\Omega}, i \in S$, with the initial condition $M_{n+1}^{i}(0, x)=0$ for $x \in \mathbb{R}^{m}$, $i \in S$.

Now, let us estimate the solution of this comparison system. Lemma 1 , Lemma 3 and the properties of $\phi$ yield

$$
\begin{aligned}
& M_{n+1}^{i}(t, x) \leq \int_{0 \mathbb{R}^{m}}^{t} \Gamma^{i}(t, x ; \tau, \xi) f^{i}(\tau, \xi) d \xi d \tau \\
& \leq \int_{0 \mathbb{R}^{m}}^{t} \frac{N_{0}}{n !}\left(L_{1}+L_{2}\right)^{n+1} \tau^{n}[\phi(\tau)]^{m / 2} \exp \left(\phi(\tau)|\xi|^{2}\right) c(t-\tau)^{-m / 2} \\
& \leq c \frac{N_{0}}{n !}\left(L_{1}+L_{2}\right)^{n+1} \int_{0}^{t} \tau^{n}[\phi(\tau)]^{m / 2}(t-\tau)^{-m / 2} \\
& \leq c \frac{N_{0}}{n !}\left(L_{1}+L_{2}\right)^{n+1} \int_{0}^{t} \tau^{n}\left(\frac{\mu^{*}|x-\xi|^{2}}{4(t-\tau)}\right) d \xi d \tau \\
&\left.\left.\times \frac{4 \pi(t-\tau)}{\mu^{*}-4 \phi(\tau)(t-\tau)}\right)^{m / 2} \quad \frac{\mu^{*} \phi(\tau)|x|^{2}}{\mu^{*}-4 \phi(\tau)(t-\tau)}\right) d \tau \\
& \leq N_{0} \frac{t^{n+1}}{(n+1) !}\left(L_{1}+L_{2}\right)^{n+1}[\phi(t)]^{m / 2} \exp \left(\phi(t)|x|^{2}\right) . \\
& \times \exp \left(\frac{\mu^{*} \phi(\tau)|x|^{2}}{\mu^{*}-4 \phi(\tau)(t-\tau)}\right) d \tau
\end{aligned}
$$


Therefore, owing to Proposition 3 we get

$$
\begin{aligned}
m_{n+1}^{i}(t, x) \leq M_{n+1}^{i}(t, x) \leq N_{0} \frac{\left[\left(L_{1}+L_{2}\right) t\right]^{n+1}}{(n+1) !}[\phi(t)]^{m / 2} & \exp \left(\phi(t)|x|^{2}\right), \\
& \text { for }(t, x) \in \bar{\Omega}, i \in S,
\end{aligned}
$$

so, the induction step is proved. Thus, $\left\|m_{n}\right\|_{2, \phi}=\left\|\widetilde{m}_{n}\right\|_{0} \rightarrow 0$ as $n \rightarrow \infty$ where

$$
\begin{aligned}
\widetilde{m}_{n} & =\widetilde{w}_{n}-\widetilde{v}_{n}, \\
\widetilde{v}_{n} & =v_{n}[\phi(t)]^{-m / 2} \exp \left(-\phi(t)|x|^{2}\right), \\
\widetilde{w}_{n} & =w_{n}[\phi(t)]^{-m / 2} \exp \left(-\phi(t)|x|^{2}\right) .
\end{aligned}
$$

Therefore, as in the proof of Theorem 1 we conclude that $\widetilde{u}:=\lim _{n \rightarrow \infty} \widetilde{v}_{n}$ is continuous and consequently so is $u=\widetilde{u} \exp \left(\phi(t)|x|^{2}\right)$.

To end the proof it is enough to repeat the proof of step (v) of Theorem 1. Finally, the uniqueness of the solution is a direct consequence of Proposition 3.

\section{References}

[1] A. Bartłomiejczyk and H. Leszczyński, Comparison principles for parabolic differential-functional initial-value problems, Nonlinear Anal. 57 (2004), 63-84.

[2] S. Brzychczy, Some variant of iteration method for infinite systems of parabolic differential-functional equations, Opuscula Math. 20 (2000), 41-50.

[3] -, Existence and uniqueness of solutions of infinite systems of semilinear parabolic differential-functional equations in arbitrary domains in ordered Banach spaces, Math. Comput. Modelling 36 (2002), 1183-1192.

[4] A. Bychowska and H. Leszczyński, Parabolic equations with functional dependence, Z. Anal. Anwend. 20 (2001), 115-130.

[5] S. D. Eidel'man, Parabolic Systems, North-Holland, 1969.

[6] A. Friedman, Partial Differential Equations of Parabolic Type, Prentice-Hall, Englewood Cliffs, NJ, 1964.

[7] G. S. Ladde, V. Lakshmikantham and A. S. Vatsala, Monotone Iteration Techniques for Nonlinear Differential Equations, Monogr. Adv. Texts Surveys Pure Appl. Math. 27, Boston, Pitman, 1985.

[8] C. V. Pao, Coupled nonlinear parabolic systems with time delays, J. Math. Anal. Appl. 196 (1995), 237-265.

[9] A. Pudełko, Existence of solutions of the Cauchy problem for semilinear infinite systems of parabolic differential-functional equations, Acta Math. Univ. Iagel. 42 (2004), 149-169.

[10] -, Existence of solutions for infinite systems of parabolic equations with functional dependence, Ann. Polon. Math. 86 (2005), 123-135.

[11] J. Szarski, Differential Inequalities, Monogr. Mat. 43, PWN, Warszawa, 1965. 
[12] J. Wu, Theory and Applications of Partial Functional Equations, Springer, New York, 1996.

Faculty of Applied Mathematics

AGH University of Science and Technology

Al. Mickiewicza 30

30-059 Kraków, Poland

E-mail: fronczyk@wms.mat.agh.edu.pl

Received 2.8.2005

and in final form 4.10.2006 